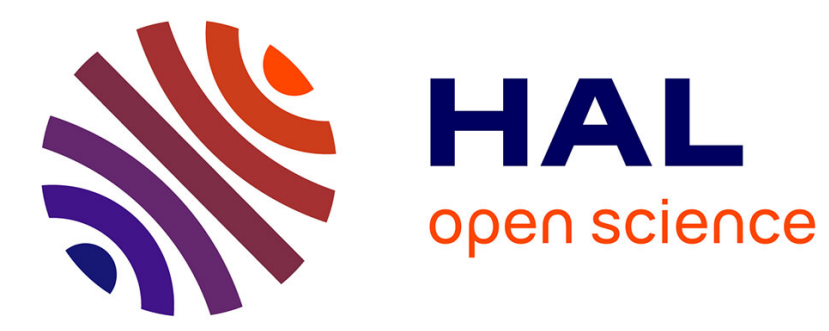

\title{
Je-sais-quoi : la représentation des formes du vivant dans l'œuvre de William Hogarth
}

Frédéric Ogée

\section{To cite this version:}

Frédéric Ogée. Je-sais-quoi : la représentation des formes du vivant dans l'œuvre de William Hogarth. 1999. hal-01576938

\author{
HAL Id: hal-01576938 \\ https://hal.science/hal-01576938
}

Submitted on 23 Sep 2017

HAL is a multi-disciplinary open access archive for the deposit and dissemination of scientific research documents, whether they are published or not. The documents may come from teaching and research institutions in France or abroad, or from public or private research centers.
L'archive ouverte pluridisciplinaire HAL, est destinée au dépôt et à la diffusion de documents scientifiques de niveau recherche, publiés ou non, émanant des établissements d'enseignement et de recherche français ou étrangers, des laboratoires publics ou privés. 


\title{
Je-sais-quoi : la représentation des formes du vivant dans l'œuvre de William Hogarth
}

\section{Frédéric Ogée}

\begin{abstract}
Frédéric Ogée : Je-sais-quoi. The representation of forms of life in the works of William Hogarth.

The rise of English painting and, more widely, of a visual culture in 18th-century Britain, is a remarkable manifestation of the new epistemology which, under the influence of Locke, Newton and the natural philosophers of the Royal Society, transformed the way the British perceived nature. The painter and engraver William Hogarth played a crucial role in this (re)volution. His works can be read as examples of the new 'observational' attitude, of the 'modern' modes of representation of natural phenomena. One of his most original characteristics — from which both his success and his difficulties with academic orthodoxy derived was the attention he paid to the variety of forms of life and the way he incorporated and played upon the irregular, the proliferating and the disorderly in nature. Both instinctively and culturally empirical, he devised an aesthetics that could account for evolution and accumulation, and new forms of representation of reality which daringly describe examples of proliferation and de-composition, turning his pictures into tangible visual experiments.
\end{abstract}

\section{Citer ce document / Cite this document :}

Ogée Frédéric. Je-sais-quoi : la représentation des formes du vivant dans l'œuvre de William Hogarth. In: Dix-huitième Siècle, n³1, 1999. Mouvement des sciences et esthétique(s) pp. 249-268;

http://www.persee.fr/doc/dhs_0070-6760_1999_num_31_1_2295

Document généré le 24/06/2016 


\section{JE-SAIS-QUOI : LA REPRÉSENTATION DES FORMES DU VIVANT DANS L'EUUVR DE WILLIAM HOGARTH}

La naissance de la peinture anglaise, et plus largement l'émergence d'une culture visuelle en Grande-Bretagne au $18^{\mathrm{e}}$ siècle, est l'une des traces les plus remarquables de la nouvelle épistémologie qui, sous l'influence d'écrits, philosophiques comme ceux de Locke ou scientifiques comme ceux de la Société Royale de Londres ou de Newton, a transformé le regard que les Anglais portaient sur le monde, à mesure que celui-ci devenait leur jardin.

Essentiel dans cette évolution est le rôle qu'a joué le peintre et graveur William Hogarth (1697-1764), dont les œuvres sont autant de témoignages des nouvelles pratiques d'observation de la nature (y compris la nature humaine) qui caractérisent cet état d'esprit. Outre le fait que ses images se présentent nettement comme des représentations de la modernité de son temps, on remarque de prime abord qu'elles se caractérisent par la présence constante d'objets et de détails concrets, dénotant un intérêt particulier et nouveau pour la matérialité de l'existence, donnée ici à voir de façon très tangible. Mais le premier effet de bric-àbrac visuel (qu'on lui reproche parfois) se révèle en fait être au service d'un véritable projet artistique, et l'une des qualités les plus originales de son œuvre, à la source tant de son succès public immédiat que de ses difficultés avec l'orthodoxie académique, est l'attention qu'il porte à la variété des formes du vivant, et la façon dont il intègre ou exploite l'irrégulier, le rugueux, le foisonnant ou le désordonné dans la Nature. Par sa démarche artistique, il cherche à rendre compte notamment de phénomènes très physiques d'évolution, de "progress », d'accumulation (dont on voit bien la façon dont ils ont pu être aussi thématisés à l'appui d'un message satirique et/ou moral), et les nouvelles formes de représentation du réel qu'il propose (désordre visuel ou séries d'images) décrivent, autant qu'elles utilisent, des "expériences » de prolifération et de dé-composition, analysées de façon très scientifique dans une dynamique toute newtonienne de causes et d'effets. En fait, lue dans une perspective plus vaste d'histoire 
des idées, on constate à quel point sa vision du monde est en phase avec les postulats de la philosophie empiriste et les convictions des nouvelles classes moyennes britanniques, convaincues des bienfaits éthiques, scientifiques, sociaux et esthétiques de la notion d'expérience. Les œuvres de Hogarth proposent autant de parcours expérimentaux fondés sur une « re-présentation », chaque fois réactivée pour et par le «spectateur» ainsi invité à acquérir, au moyen de l'image, une véritable connaissance des formes du vivant dans leur variété dynamique.

La matérialité à l'œuvre dans les images de Hogarth se perçoit d'abord dans les sujets qu'il représente. Adoptant à son tour l'attitude «spectatoriale » que promouvaient les périodiques en vogue du début du siècle ${ }^{1}$, il porte son regard sur les diverses facettes de la réalité contemporaine, seul domaine pertinent d'un art « moderne » selon lui. Qu'il réalise des portraits de commandc de notables en famille ou des scènes de genre à visée moralisatrice, ses images, toujours, visent à saisir la réalité dans son mouvement et sa particularité concrète, avec cette "inlassable vigilance » qui constituait, pour les fondateurs de la «Royal Society of London for the Improving of Natural Knowledge » (qui reçut sa charte royale en 1662-3), la qualité primordiale du «philosophe expérimental »?

Ses portraits sont des « conversation pieces » où l'identité des modèles, abandonnant la pose et le vêtement d'apparat, émerge d'une représentation de relations sociales plurielles, inscrites dans un environnement quotidien fait d'animaux, de mobilier, d'objets utilitaires ou décoratifs, qui se combinent pour représenter au spectateur un milieu vivant [FIG. 1]. De même, dans les scènes de genre, rejetant tout principe de sélectivité académique (unité d'action) ou d'abstraction esthétisante (c'est ce que lui reprochera Reynolds ${ }^{3}$ ), il plonge le spectateur dans un spectacle «total»

1. On pense surtout à l'influence déterminante du bien nommé Spectator (1711-14) de Addison et Steele.

2. «[...] these Studies which require such an indefatigable watchfulness », (Thomas Sprat, The History of the Royal Society (London, 1667), p. 337) [ma traduction!.

3. Particulièrement dans les trois articles qu'il envoya entre septembre et novembre 1759 au périodique The Idle'r de Samuel Johnson. Au coeur du débat qui faisait alors rage sur l'opportunité de créer à Londres une Académie Royale de peinture sur le modèle français. Reynolds dénonce "cette sorte d’imitation » à laquelle il préfère "le grand style". Pour lui, un art fondé sur la scule observation ne saurait exprimer « les idées invariables, nobles et générales $[\ldots]$ inhérentes à la nature universelle» car « en peinture, comme en poésie, le style le plus haut est celui qui comporte le moins d'éléments de la nature commune » (Joshua Reynolds, Letter n" 79 to «The Idler » in the Universal Chronicle (20 oct. 1759), p. 319 [ma traduction]. 
où le regard est invité à refaire visuellement l'expérience d'une réalité brute (et parfois brutale), et à observer, tel le savant, les nombreuses facettes contiguës de l'interaction dynamique entre l'être humain et son environnement naturel et matériel. Avant d'y lire quelque message satirique ou moral, on est immédiatement confronté dans ces images à la matérialité visuelle, sonore, tactile du réel [FIG. $2-3]^{4}$. Le rigide et le sinueux, le solide et le liquide, le clair et le sombre, le fragile et le solide, le dur et le mou, etc., les images de Hogarth offrent des notations très précises de tous ces paramètres physiques du réel, qui deviennent les indices d'une nouvelle conception de la représentation artistique, désormais conçue comme une vraie expérience visuelle, au sens

4. Un ouvrage récent sur Hogarth est consacré intégralement à la précision des représentations d'objets en porcelaine dans ses images, montrant notamment à quel point celles-ci sont en phase avec les développements importants qui marquèrent la production de céramique en Angleterre : Lars Tharp, Hogarth's China: Hogarth's Paintings and 18th-Century Ceramics (Londres, 1997). 
expérimental du terme, et qui constitueront, en contrepoint aux icônes de l'art antique, l'alphabet formel de son traité d'esthétique, L'Analyse de la Beauté, publié en 1753 [FIG. 4] ${ }^{5}$. Comme le dit Thomas Sprat, dans l'Histoire de la Société Royale (The History of the Royal Society, Londres, 1667), l'expérimentateur " regarde chaque chose à son niveau, et non d'un point de vue supérieur : il s'intéresse aux objets bruts et non classifiés ("undigested") de ses sens, sans les considérer comme ils peuvent l'être quand on les réunit sous forme de notions. Il a la possibilité de comprendre les façons les plus naturelles dont les choses sont produites. Il perçoit clairement tous les accidents et les bifurcations, tous les avantages et les défauts secrets de la Nature. Il s'efforce de savoir plutôt que d'admirer ${ }^{6}$.

Cette volonté de comprendre, et à terme de faire comprendre en re-présentant, s'exprime chez Hogarth par la manière dont il a tenté, là encore de façon expérimentale, de mettre en forme dynamique et artistique le matériau brut dont il se saisissait au moyen d'une remarquable mémoire visuelle, afin d'en proposer des images « révélatrices » permettant de «savoir», après des décennies au cours desquelles l'Angleterre s'était déchirée autour de la façon de bien « admirer ». L'influence manifeste des idées empiristes sur sa pratique et ses théories, particulièrement les écrits de Locke, a été analysée dans plusieurs études récentes ${ }^{7}$. Mais ce qui frappe aussi, en rapport avec le thème de ce numéro de Dix-Huitième Siècle, c'est à quel point les formes originales

5. Thomas Sprat, parlant du nouvel intérêt des savants pour le grand livre de la Nature, parle de «tout cet alphabet de la Nature, qui se représente à notre considération en tant de volumes infinis » [ « the whole Alphabet of Nature, which represents itself to our Consideration, in so many infinit [sic] Volumes », History, p.345].

6. «The Experimenter [...] looks on every thing standing equal to it, and not as from a higher ground: He labors about the plain and undigested objects of his senses, without considering them as they are joyn'd into common Notions. He has an opportunity of understanding the most natural ways by which all things are produc'd. He cleerly [sic] beholds all the secret accidents and turnings. advantages and failings of Nature. He indevors rather to know, than to admire » (Sprat, History, p. 334-5) [ma traduction].

7. Voir en particulier l'article de Michel Baridon, "Hogarth the empiricist ", ainsi que mon "Aesthetics and empiricism : the ideological context of Hogarth"s series of pictures ", dans The Dumb Show: image and society in the works of William Hogarth, Frédéric Ogéc, ed., Studies on Voltaire... (1997), vol. 357, p. 191-202; p.177-189. Voir aussi le remarquable catalogue de l'exposition Hogarth and his times, réalisé par David Bindman (Londres, 1997), en particulier le chapitre 5, «Disenchantment: Hogarth, God and Science » (p. 51-57). 


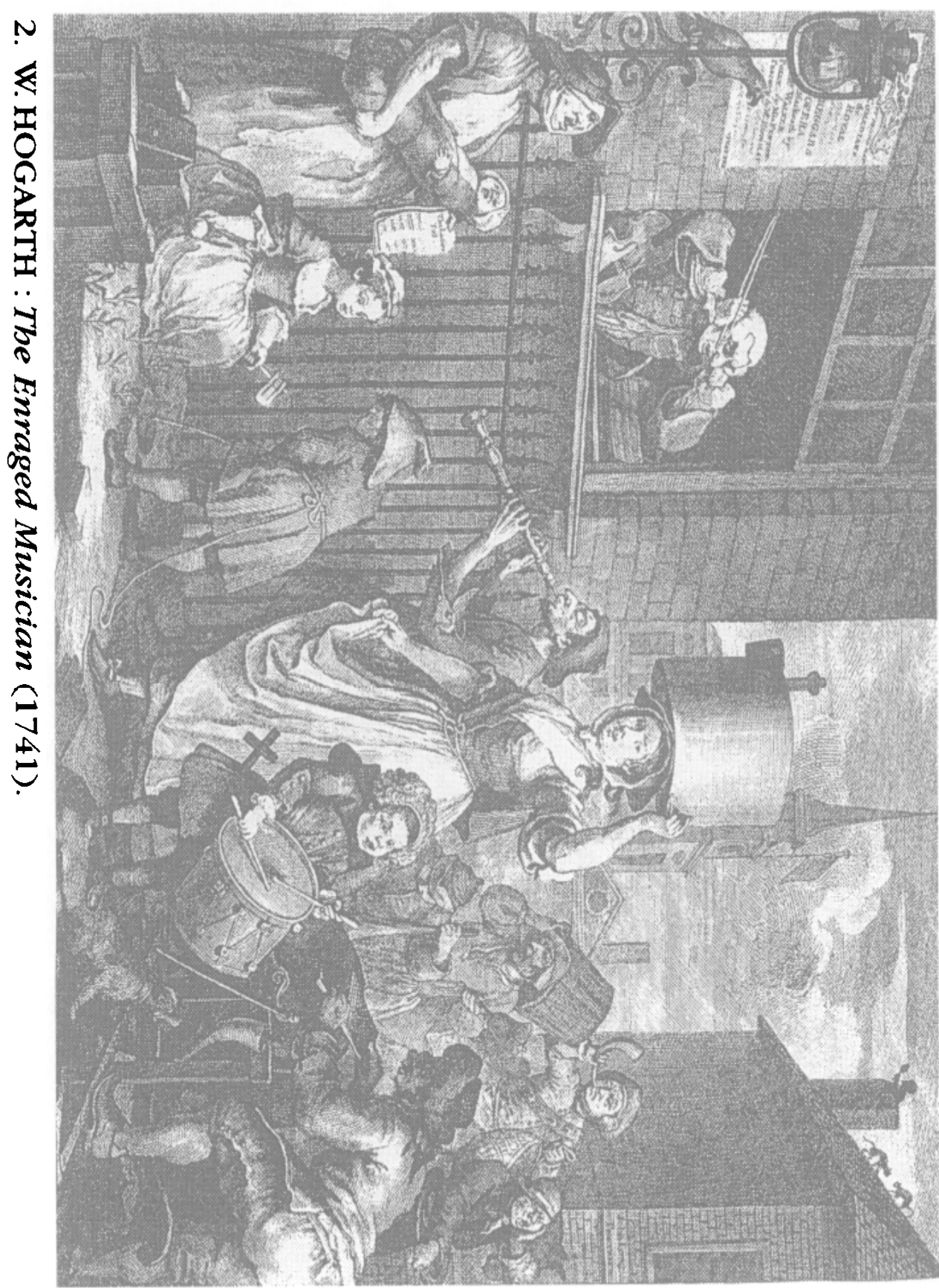

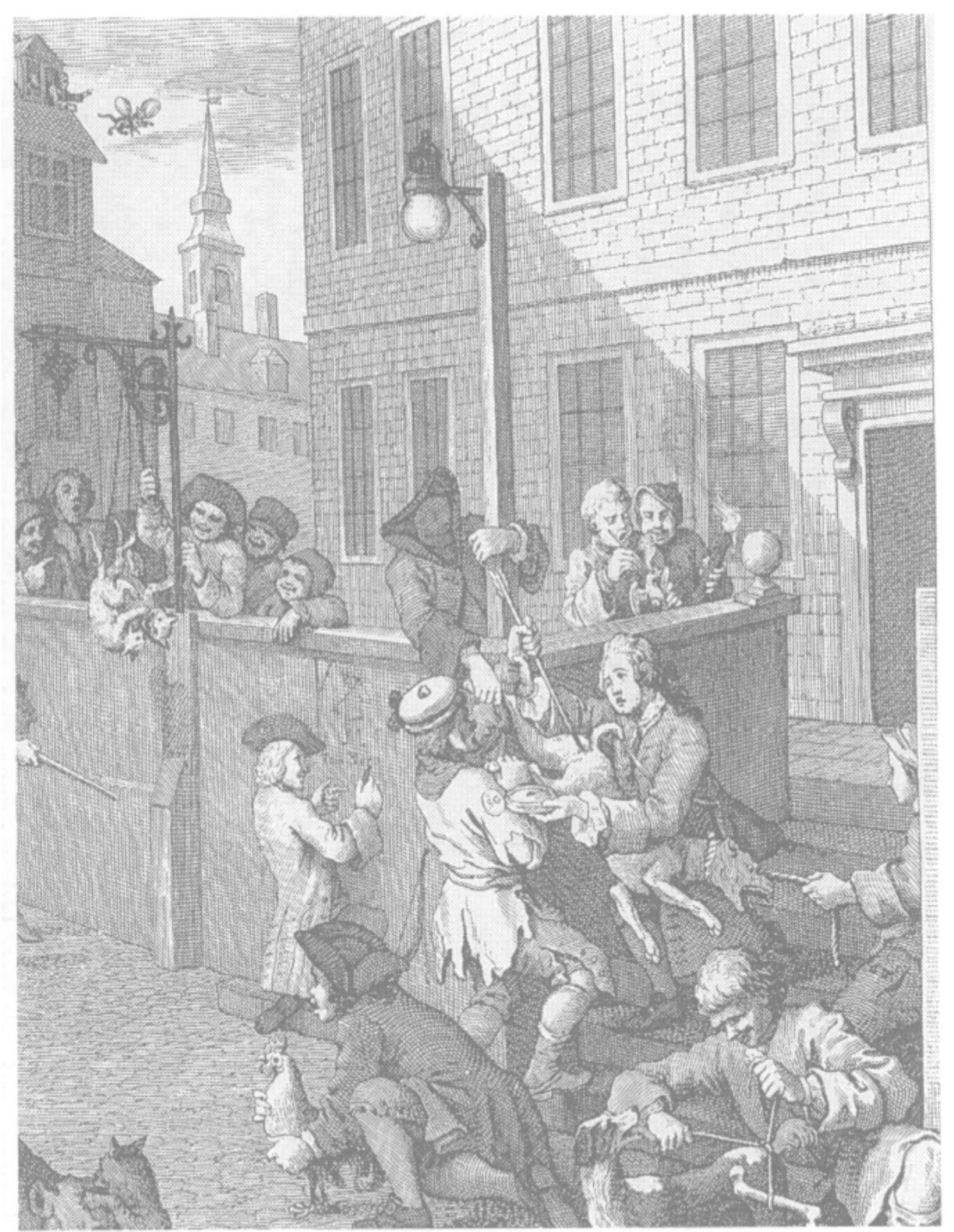

3. W. HOGARTH : The First Stage of Cruelty (1751). 
qu'a prises son art - particulièrement le principe de la série d'images - dénotent une démarche en bien des points comparable à celle de savants comme Robert Hooke (1635-1703), l'auteur du célèbre Micrographia; or some Physiological Descriptions of Minute Bodies made by Magnifying Glasses (Londres, 1665), et l'un des piliers de la Royal Society à ses débuts, lui aussi fervent défenseur des vertus épistémologiques de l'image.

Hooke, dont l'importance a quelque peu été éclipsée par la controverse fameuse qui l'opposa à Newton, était le responsable des expériences («Curator of Experiments») à la Royal Society. Lui-même expérimentateur infatigable, il consacra une partie importante de son travail à déterminer (et réaliser) les transcriptions, les représentations les plus fidèles de ses observations, selon une formule célèbre, « une main sincère et un œil fidèle, pour examiner et enregistrer les choses elles-mêmes comme elles apparaissent ${ }^{8}$.

Porte-parole efficace de la démarche scientifique mise en avant par les nouveaux savants, sous l'influence notamment des écrits de Bacon, il cherche à libérer l'étude de la Nature des différents fardeaux qui, selon lui, entravent sa progression, freinent l'acquisition de la connaissance et ne permettent pas l'émergence d'une « nouvelle science anglaise » (on reviendra plus loin sur cette composante nationaliste). Qu'il s'agisse de l'héritage encombrant des Anciens, des «chimères » de la philosophie spéculative ou de l'obscurantisme religieux, Hooke pense que «la science de la Nature est depuis trop longtemps sous l'emprise exclusive de l'esprit et de l'imagination : il est maintenant grand temps qu'elle revienne à la simplicité et la sûreté d'observations des choses matérielles et évidentes ${ }^{9}$.

8. «A sincere hand and a faithful eye, to examine, and to record, the things themselves as they appear » (Hooke, Micrographia; or some Physiological Descriptions of Minute Bodies made by Magnifying Glasses. Londres, 1665, The Preface, s.p. [ma traduction]), formule à laquelle fait écho l'Ode du poète Abraham Cowley placée en exergue de la History de Sprat : «He before his sight must place / The Natural and Living Face;/ The real object must command / Each Judgment of his Eye, and Motion of his Hand »]. C'est également le titre judicieusement choisi par Svetlana Alpers pour l'un des chapitres de son Art of Describing: Dutch Art in the Seventeenth Century (Chicago, 1983).

9. «The truth is, the science of nature has been already too long made only a work of the brain and the fancy: It is now high time that it should return to the plainness and soundness of Observations on material and obvious things " (Micrographia, Preface, [ma traduction]). 


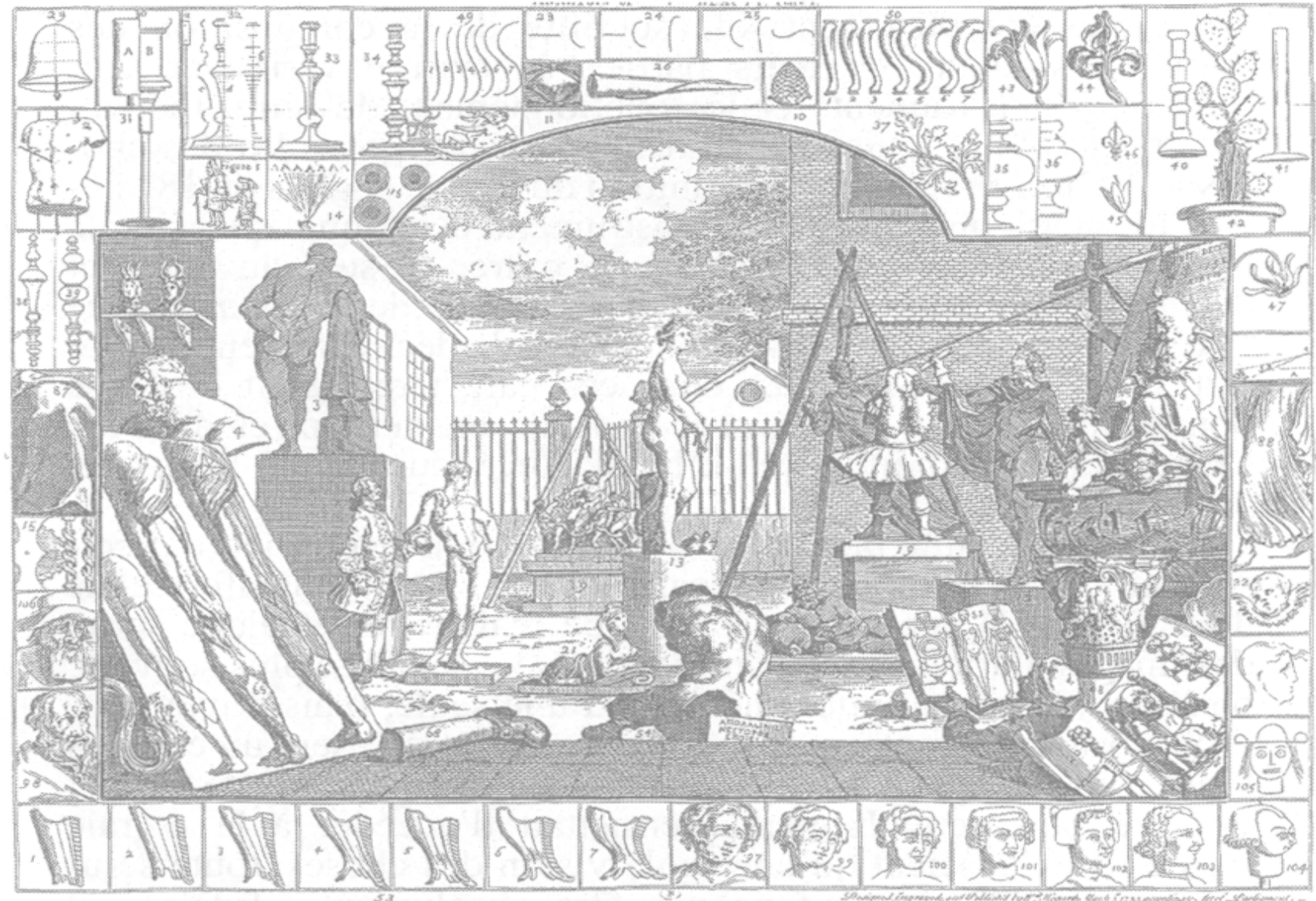

4. W. HOGARTH : L'Analyse de la Beauté (1751), planche 1.

Convaincu de l'intérêt des possibilités offertes par les nouveaux instruments d'optique que sont le microscope et le télescope, il y voit la possibilité d'améliorer la simple appréhension du monde par les sens, en " suppléant à leurs infirmités grâce à des instruments et, en quelque sorte, en ajoutant des organes artificiels à ceux de la Nature ». Grâce à cela, l'homme aura accès à un «nouveau monde visible ${ }^{10}$.

En publiant ses Micrographia en 1665, il a voulu montrer en quoi l'image grossie du spectacle de la Nature pouvait être en quclquc sorte « correctrice ", puisqu'elle opère une amélioration de la vue. Le recours à des instruments d'optique, qui multiplient les ressources de l'observation de la nature, permet de «décou-

10. "The next care to be taken, in respect of the senses, is a supplying of their infirmities with instruments, and, as it were, the adding of artificial organs to the natural. $[. .$.$] ; hence there is a new visible world discovered to the understan-$ ding » (Micrographia, Preface [ma traduction]). 
vrir » plus complètement «la subtilité de la composition des corps, la structure de leurs parties, la texture variée de leur matière, les instruments et le fonctionnement de leurs mouvements internes et toutes les autres apparences possibles des choses " $"$ ". On croirait lire une définition du roman anglais du $18^{\circ}$ siècle, lui aussi bien davantage instrument d'optique que simple miroir prétendument « réaliste ». En outre, au sein du détail et de l'infiniment petit se trouve tout un monde de la connaissance fait, peut-être, de signes divins en attente de déchiffrement : «Qui sait si le Créateur n'a pas, dans ces caractères, écrit et gravé bon nombre de ses desseins et conseils mystérieux et donné à l'homme la possibilité, avec diligence et industrie, de les lire et de les comprendre ? $\gg{ }^{12}$

Mais l'originalité principale de Hooke, dont on trouvera précisément l'équivalent chez Hogarth, s'est surtout manifestée de deux façons. D'une part, il a tenté de réaliser, en quelque sorte, des images plurielles, ses « micrographies » étant le plus souvent, on le sait, la transcription, non pas d'une seule, mais d'une suite d'observations. Hooke avait constaté en effet que seul un examen répété, en variant le jeu de la lumière et la position de l'objet observé dans cette lumière, permettait d'accéder à la « vraie forme $"{ }^{13}$. Conscient qu'une seule vision des choses, toute scrupuleuse fût-elle, pouvait ne pas être absolument «fidèle », il multipliait les observations afin de bien se saisir de toutes les facettes du réel, avant d'en proposer une image « de synthèse » dont le formidable paradoxe est d'être d'autant plus «vraie » qu'elle est fictive. Anticipant de façon remarquable le principe de modélisation possible de nos jours grâce à l'image virtuelle (elle aussi appelée image de synthèse), Hooke désigne par sa pratique de la représentation tout un champ d'activité de la « fic-

11. «By these helps the subtility of the composition of Bodies, the structure of their parts, the various texture of their matter, the instruments and manner of their inward motions, and all the other possible appearances of things, may come to be more fully discovered » (Micrographia, Preface [ma traduction]).

12. "Who knows, but the Creator may, in those characters, have written and engraven many of his most mysterious designs and counsels, and given man a capacity, which, assisted with diligence and industry, may be able to read and understand them » (Observation XXIX Of the Seeds of Tyme, Micrographia, p. 154 [ma traduction]).

13. «There is much difficulty to discover the true shape [of microscopic objects], the same Object secming quite differing, in one position to the Light, from what it really is, and may be discover'd in another. And therefore I never began to make any draught before by many examinations in several lights, and in several positions to those lights, I had discover'd the true form " (Micrographia, Preface [ma traduction]). 
tion », non plus comme «mensonge » (c'est le sens du mot au début du $18^{\mathrm{e}}$ siècle) mais comme transcription "intensifiée »du réel rendue possible par le recours à des instruments d'optique, dont toute l' avant-garde artistique anglaise du $18^{\mathrm{e}}$ siècle, du jardin au roman en passant par la peinture, exploitera l'idée ${ }^{14}$.

D'autre part, Hooke a défini le travail d'observation et d'analyse comme un processus circulatoire d'échanges incessants, partant de l'appréhension sensorielle du réel et de l'acquisition de données pour se poursuivre avec le traitement de celles-ci par la mémoire puis leur interprétation par la raison, avant de revenir au réel pour de nouvelles confrontations expérimentales ${ }^{15}$. $\mathrm{Au}$ cour de ce processus, l'image «enrichie» de Hooke joue un rôle charnière crucial puisqu'elle permet de (se) représenter un réel "plus vrai que vrai », invitant donc à une expérience de perception dynamique (une «re-présentation») au cours de laquelle le spectateur « découvre » un monde tout à la fois même et autre, connu et nouveau, et qu'il apprend à mieux connaître grâce à cette tension entre écart «éclairant » de la représentation et norme appréhensible par les sens (en temps «normal»). L'image acquérait ainsi un statut épistémologique inédit, non plus illustration ou imitation, mais mise en tension du réel de façon à éprouver les limites de l'observation première, de certifier les conditions d'une bonne vision en cherchant la « vraie forme » au travers d'une expérience constamment renouvelée, littéralement une expérience d'une autre Nature.

C'est exactement selon les mêmes principes, et parfois dans les mêmes termes, que Hogarth fit évoluer son art. Ses deux

14. Sur cette notion de "modélisation » dans la fiction anglaise du $18^{\mathrm{e}}$ siècle, voir aussi l'ouvrage de James Thompson, Models of Value. Eighteenth-Century Political Economy and the Novel (Duke University Press, 1996). Notons que l'enthousiasme optimiste des fondateurs de la Royal Society pour les nouvelles possibilités du microscope et du télescope, autorisant selon eux une vue plus juste et plus précise, avait fait immédiatement l'objet de critiques, particulièrement celles de Henry Stubbe qui, tout en étant un partisan convaincu de la méthode empirique, refusait d'y voir une nouvelle religion irréfutable, et critiqua les "expérimentateurs » de la Royal Society, qu'il appelait, de façon tout à fait intéressante quand on sait la fortune ultérieure de ce mot, les « Novellists».

15. "So many are the links, upon which the true philosophy depends, of which, if any one be loose, or weak, the whole chain is in danger of being dissolved; it is to begin with the hands and eyes, and to proceed on through the memory, to be continued by the reason; nor is it to stop there, but to come about to the hands and eyes again, and so, by a continual passage round from one Faculty to another, it is to be maintained in life and strength, as much as the body of man is by the circulation of the blood through the several parts of the body » (Micrographia, Preface). 
principales «expérimentations » en matière d'image ont visé, elles aussi, à proposer des représentations «totales» du réel, dont la perception serait ainsi enrichie par l'optique de l'art, par l'artifice.

D'abord, un peu à la manière d'un Hooke, il a conçu des images plurielles en ce qu'elles tentent de rendre compte simultanément d'événements successifs dans le temps ou dans l'espace. Ainsi, dans A Midnight Modern Conversation (1733) [FIG. 5], il représente de manière contiguë (série de personnages) et composée (autour d'une même table) les différents effets de la boisson, les différents stades de l'ébriété (gaîté, vocifération, vacillement, hébétude, vomissement, inconscience, chute). Cette image, l'une des plus célèbres de Hogarth ${ }^{16}$, offre un excellent exemple du travail pictural de Hogarth sur la matérialité du réel. Comme je l'ai analysé plus en détail ailleurs ${ }^{17}$, clle tente à la fois de représenter et de faire ressentir les effets de l'alcool grâce à une double dynamique. Dynamique de texture d'abord, faite d'une friction permanente du mou et du rigide, du sinueux et du rectligne, invitant à un parcours oculaire aussi étourdissant que la boisson, à une perception déstabilisée, presque nauséeuse, au contact des reliefs et des soubresauts de cette "conversation ": à la pureté et la simplicité des lignes du décor et des objets s'oppose le désordre des «traits » humains. Dynamique temporelle ensuite, puisqu'en juxtaposant les différents moments de l'ivresse, bornés par les traces du passé (bouteilles vides) et les présages du futur (la manche qui s'enflamme), Hogarth a tenté de restituer le déroulement, le «progress » de l'expérience en une image unique, somme visuelle synthétique des causes et des effets, donnant à voir un réel intensifié.

De façon semblable, dans ses nombreuses scènes de foule [FIG. 6] ${ }^{18}$, il a représenté des effets saisissants de prolifération,

16. Lars Tharp (ouvrage cité, p.46-55) analyse le remarquable rayonnement international de cette image, dont le thème se prêtait bien à l'ornementation de pièces en porcelaine.

17. Frédéric: Ogée, «I 'onction extrême : une lecture de A Midnight Modern Conversation (1733) de William Hogarth », Études Anglaises 45.1 (1992), p. 5665. Dans cet article, je tente également de prendre en charge l'allusion iconographique au motif de la Cène qui structure cette image, pour proposer une lecture de celle-ci en termes de blasphème, la boisson étant ici servie par un ecclésiastique et les gestes sacramentels du baptême et de l'eucharistie apparaissant aussi clairement.

18. Voir aussi les planches 11 et 12 de Industry \& Idleness (1747), The March to Finchley (1750), les planches 1 et 4 de An Election: Four Pictures (1757) ou Credulity. Superstition, and Fanaticism (1762). 


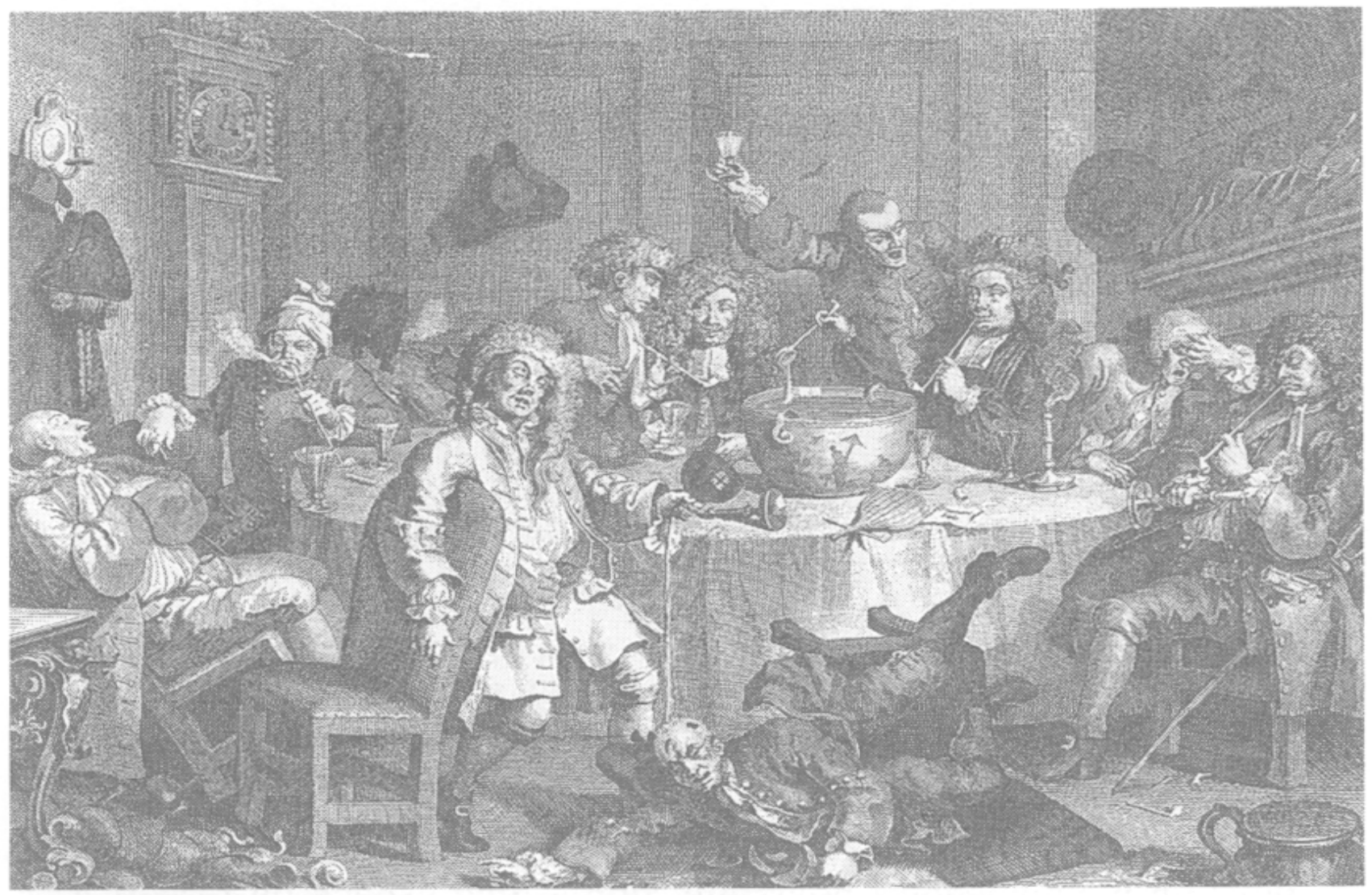

5. W. HOGARTH : A Midnight Modern Conversation (1733).

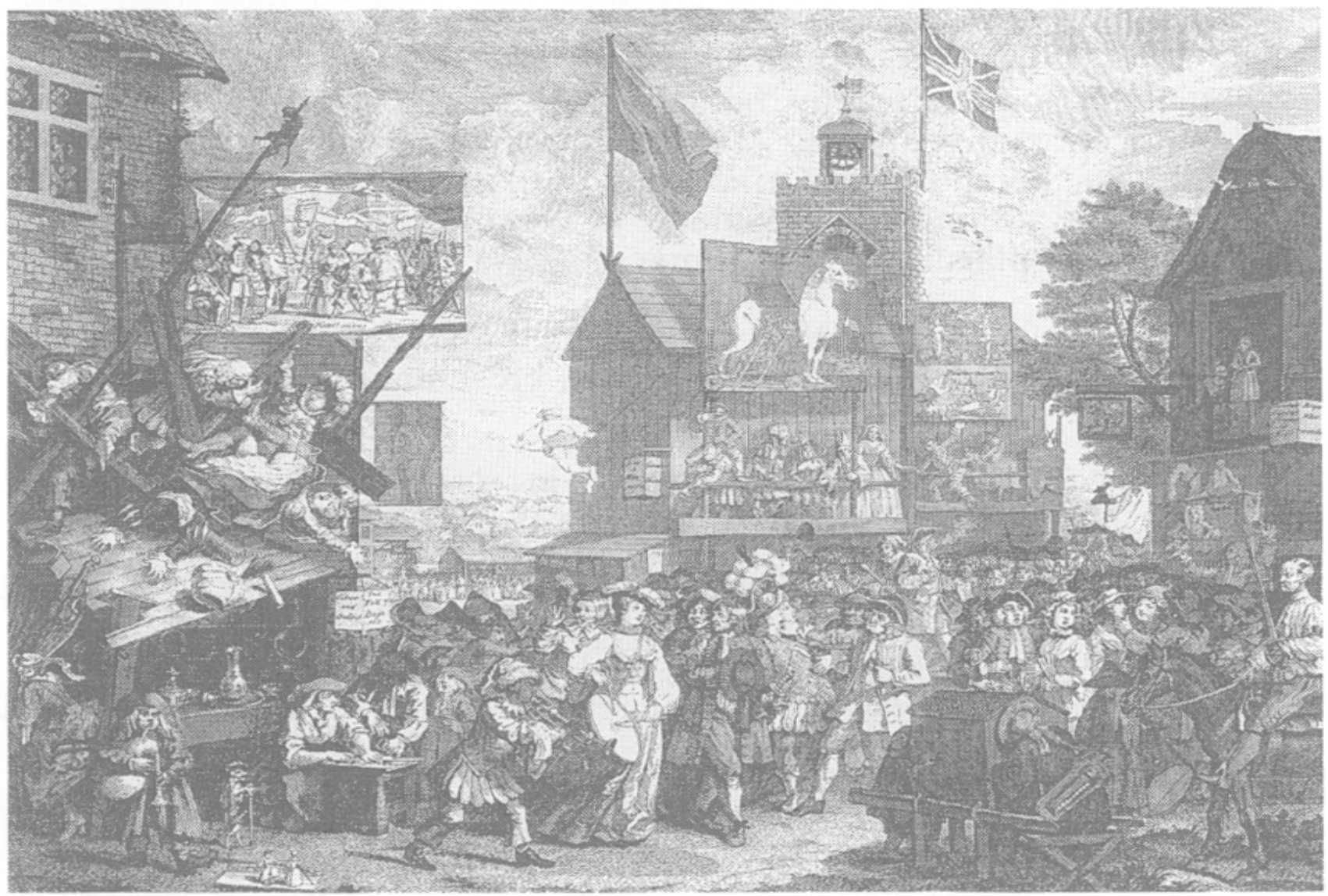

6. W. HOGARTH : Soutbwark Fair (1733 - 1734). 
utilisant toute l'énergie de texture offerte par le matériau humain et le jeu social, observant, tantôt avec la froideur de l'anatomiste tantôt avec la méticulosité de l'entomologiste, l'extraordinaire variété du réel et la «spontanéité » des gangrènes et tumeurs qui peuvent en démultiplier les cellules malignes ${ }^{19}$.

$L$ 'autre innovation ou expérimentation formelle de Hogarth ${ }^{20}$, elle aussi destinée à faire percevoir le réel de façon «enrichie », a été de déployer sa représentation sur plusieurs images, offrant ainsi au spectateur un parcours visuel dont le but est, là encore, de faire prendre conscience des différents stades ou aspects de l'expérience de ce réel, et de percevoir «tous les accidents et bifurcations » de la Nature dont parlait Sprat. Ses grandes séries narratives (Harlot's Progress, Rake's Progress, Marriage à-laMode, Industry \& Idleness) ou purement séquentielles (The Four Times of the Day, The Four Stages of Cruelty, An Election: Four Pictures) offrent au regard une multitude de parcours exploratoires, à la source, selon lui, de l'émergence de la beauté : «Poursuivre est l'occupation de notre vie entière, et nous cause du plaisir [...]. Toute difficulté qui se présente et qui interrompt pour un moment notre poursuite, donne une espèce de ressort à l'esprit, varie nos plaisirs, et fait un amusement et une jouissance de ce qui, sans cela, aurait causé de la peine et de l'effort. [...] L'œil jouit de cette sorte de plaisir en suivant des sentiers tortueux, des rivières qui serpentent, et tous les autres objets dont les formes sont principalement composées de ce qu'on appelle lignes ondoyantes ou serpentines [...]. Je définis donc la complication des formes [comme étant] cette disposition des lignes qui compose la surface des corps, qui fait faire à l'œil une espèce de chasse agréable [《a wanton kind of chace »], et qui, par le plaisir qu'elle donne à l'esprit, mérite le nom de beauté » 21 .

Il s'agit pour le spectateur, au travers d'une perception toujours dynamique où s'opère le travail de re-présentation, de percevoir

19. Sur cette fascination de Hogarth pour les phénomènes d'accroissement (le mot "growth» en anglais signifie à la fois croissance et tumeur) et de prolifération, voir mon " "And Universal Darkness buries All": Hogarth and cxcess ", in The Dumb Show, ouvrage cité, p. 79-96.

20. On pourra objecter que les deux formes de représentation présentées ici comme « innovations » ont de célèbres antécédents (on pense à Bruegel l'Ancien ou à Bosch pour les effets de prolifération, aux vitraux médiévaux ou aux fresques d'un Fra Angelico pour les « histoires en série »), mais c'est de leur modernité dans les premières décennies du $18^{\mathrm{e}}$ siècle anglais, de leur nouvelle pertinence esthétique, qu'il est question ici.

21. William Hogarth, Analyse de la Beauté, destinée à fixer les idées fluctuantes que l'on a du goût (1753), trad. de Jansen (1805) (Paris, 1991), p. 66-67. 
les nombreux changements d'une image à l'autre et ainsi de "découvrir » ce que Locke appelait «la texture et le mouvement des parties les plus petites des objets corporels $" 22$. Chaque image de la série est d'une remarquable profusion visuelle, où l'œil déjà serpente entre les formes, mais la perception est aussi activée par l'énergie sérielle, qui invite à faire l'expérience du passage du temps, à en mesurer les conséquences [FIG. 7], et à fournir en fin de parcours un travail de sommation, la connaissance se révélant bien être l'aboutissement d'un complexe et sinueux parcours cognitif. Le parcours de la prostituée, de l'innocence campagnarde à la sordide mise en bière (Harlot's Progress, 1732, 6 images), celui du roué, de l'héritage impromptu à l'asile d'aliénés de Bedlam (Rake's Progress, 1735, 8 images), ceux du couple Squanderfield, marié contre son gré par des pères calculateurs, et miné par le mensonge, la maladie, là mascarade jusqu'à la mort (Marriage à-la-Mode, 1745, 6 images), toutes ces expériences humaines se décomposent, au propre comme au figuré, sous l'œil d'un spectateur atterré au bout du compte par ces "précipités " d'existences humaines ramenées à de tragiquement laconiques séries de bifurcations fatales.

Mais le regard, loin d'être le passif témoin d'un spectacle qui lui serait offert, valide l'expérience artistique en y contribuant, empiriquement, par son travail de re-composition. Le spectateur, loin d'être le récepteur distant de quelque représentation « classique $\gg$ où se jouent de hautes tragédies un peu mystérieuses et qui le dépassent, complète la re-présentation moderne à l'aune de ses propres sinuosités, s' approprie l'expérience en la re-créant, et c'est bien ce renouvellement récurrent de l'expérience, sa capacité à être réfutée ou mise à l'épreuve du vécu de chacun, qui conférera à l'œuvre ses dimensions éthique et épistémologique.

Il s'agit là d'un des postulats essentiels de la nouvelle science : la valeur de l'expérience et son propre «progrès » viennent précisément de la qualité et de la permanence d'un travail de « réfutabilité »: «Cette analyse critique réitérée [...] doit permettre de placer hors d'atteinte de toute contestation raisonnable la réalité de ces opérations que la Société [Royale] aura positivement jugées comme réussies. Si quiconque jugeait néanmoins après cela que sa juste liberté philosophique lui interdisait de faire crédit à ces conclusions, il aurait raison, et sa différence serait reçue avec

22. "The texture and motion of the minute parts of corporeal things » (John Locke, An Essay concerning Human Understanding, $4^{c}$ éd. (1700), ed. Peter H. Nidditch (Oxford, 1975), II.XXIII, p. 303 [ma traduction]). 
gratitude, si elle était fondée sur des travaux solides, et non seulement sur des préjugés ou des soupçons $»{ }^{23}$.

Cette insistance sur le rejet des «préjugés » et des «soupçons » est symptomatique de la double priorité idéologique qui a présidé à l'émergence de la nouvelle épistémologie scientifique. Après des décennies de déchirements internes (fort préjudiciables à la bonne marche des affaires anglaises) au cours desquelles les affrontements irréconciliables ont porté sur des questions «verticales » d'ordre théologique et politique, on comprend l'intérêt qu'il y a à, selon la célèbre formule de Max Weber, "désenchanter » le monde, à « abaisser le regard » afin, d'une part, de se regrouper autour d'une observation plus "horizontale ", plus scientifique et objective (et donc moins polémique) de la nature concrète environnante, et d'autre part de promouvoir la suprématie du nouveau regard anglais sur le monde ${ }^{24}$.

De nombreuses pages du texte de Sprat sont consacrées aux vertus rassembleuses, "conversationnelles », du projet de la Société Royale, et à la chance historique et géographique de l'Angleterre de faire un pas décisif et conquérant dans la modernité. A la "croisée du nord et du sud », ses ports " ouverts de tous côtés » aux échanges avec le reste du monde, le pays a été doté par la Nature d'une situation exceptionnelle qui en fait «le siège le plus adéquat pour le progrès de la connaissance » : «Sur cet avantage de notre île, il faut tellement mettre l'accent [...] que

23. "This critical, and reiterated scrutiny of those things [...] must needs put out of all reasonable dispute, the reality of these operations, which the Society shall positively determine to have succeeded. If any shall still think it a just philosophical liberty, to be jealous of resting on their credit: they are in the right ; and their dissentings will be most thankfully receiv'd, if they be establish'd on solid works, and not onely on prejudices, or suspicions " (Sprat, ouvrage cité, p. 99 [ma traduction]). C'est Karl Popper qui, dans l'une de ses études critiques de ce qu'il appelle l'observationnisme baconien, a insisté sur l'importance de ce critère de « réfutabilité » ou « falsifiabilité ». Il parle aussi de « testability »: «Observations or experiments can be accepted as supporting a theory (or a hypothesis, or a scientific assertion) only if these observations or experiments are severe tests of the theory - or, in other words, only if they result from serious attempts to refute the theory » (Karl Popper. The Myth of the Framework. In defence of science and rationality, ed. by $M$. A. Notturno, Londres et New York, 1994, p. 88-89).

24. "Pour couper court à la magie et rationaliser le mode de vie, il n'y a jamais cu à travers toutes les époques qu un seul moyen : les grandes prophéties rationnelles. [...] Ce sont [ces] prophéties qui sont parvenues à sortir le monde de la magie [Entzauberung der Welt] et qui créèrent par là même aussi les bases de notre science moderne, de la technique et du capitalisme » (Max Weber, Histoire économique. Esquisse d'une histoire universelle de l'économie ét de la société (1923), Paris, 1991, p. 379). 

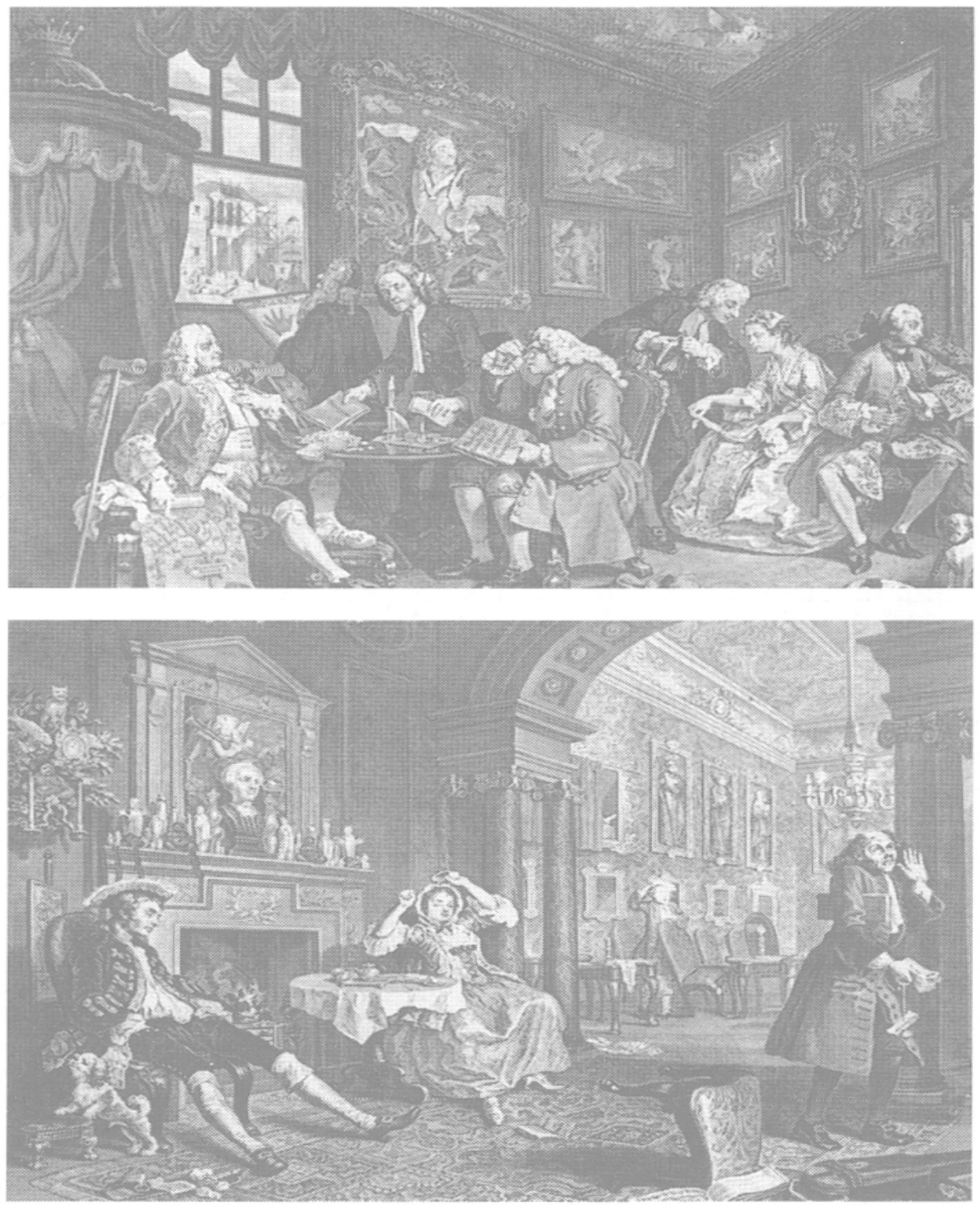

7. W. HOGARTH : Le mariage à la mode (1745). Planches 1 et 2. 
si nous devions chercher à travers le monde un lieu perpétuel d'habitation où la Philosophie universelle pourrait s'installer, aucun ne pourrait se comparer à Londres. [...] Même la position de notre climat, l'air, l'influence des cieux, la composition du sang anglais, de même que les étreintes de l'océan, semblent s'unir aux travaux de la Société Royale pour faire de notre pays une terre de connaissance expérimentale. Et c'est bien le signe que la Nature révélera davantage de ses secrets aux Anglais qu'aux autres ; parce qu'elle les a déjà dotés d'un génie si bien proportionné pour recevoir et conserver ses mystères » ${ }^{25}$.

Cette double caractéristique, conversationnelle et nationaliste en quelque sorte, fonde de façon essentielle la démarche artistique de Hogarth et la réflexion qu'il mène sur l'art. Comme l'a rappelé David Bindman, il faisait partie d'un milieu intellectuel fermement convaincu des bienfaits sociaux et moraux d'une approche scientifique de l'homme et de la nature, et rallié à l'idée (newtonienne) selon laquelle la science est en fait l'étude permettant la compréhension des signes de la présence de Dieu dans la Création, à travers l'établissement d'une sorte de généalogie des causes et des effets remontant jusqu' au primum mobile ${ }^{26}$. On a vu à quel point la grammaire compositionnelle de Hogarth trouvait sa raison d'être précisément dans la mise en relief de la causalité des comportements et des traits, prise dans son déroulement temporel. Comme le fit remarquer avec acuité William Hazlitt : «Il a représenté les mœurs et les humeurs des hommes dans l'action, et leur caractère par une expression variée. Tout dans

25. «Upon this advantage of our Island, there is so much stress to be laid, towards the prosperity of this design; that if we should search through all the world, for a perpetual habitation, wherein the universal philosophy might settle it self ; there can none be found, which is comparable to London. [...] Even the position of our climate, the air, the influence of the heaven, the composition of the English blood; as well as the embraces of the ocean, seem to joyn with the labours of the Royal Society, to render our Country, a land of experimental knowledge. And it is a good sign, that Nature will reveal more of its secrets to the English, than to others ; because it has already furnish'd them with a genius so well proportion'd, for the receiving, and retaining its mysteries " (Sprat, ouvrage cité, p.86-87; 114-115. Ma traduction).

26. Bindman, ouvr. cité, p. 51-2. Dans cet entourage, on trouve Thomas Morell (1703-84), pasteur protestant, auteur de livrets d'oratorios pour Haendel, exemple classique, selon Bindman, de ces ecclésiastiques latitudinaires tout à fait en phase avec le nouveau monde intellectuel et théologique créé par Locke et Newton. Morell aida beaucoup Hogarth pour la rédaction de son Analyse de la Beauté, lui fournissant notamment de nombreuses références classiques. On trouve aussi l'évêque Benjamin Hoadly [dont Hogarth réalisa un superbe portrait en 1741 (Tate Gallery)], newtonien convaincu, représentant très en pointe de l'aile la plus rationaliste [il fut fréquemment accusé de "déisme»] de l'Église d'Angleterre. 
ses images a de la vie et du mouvement. Non seulement l'action de la scène n'est jamais immobile, mais chaque trait est mis à totale contribution; le sentiment exact du moment est mis en évidence et porté à son degré le plus haut, puis instantanément saisi et plaqué sur la toile pour toujours. L'expression est toujours prise en passant, à un stade de sa progression ou de ses changements, et, pour ainsi dire, au moment saillant ${ }^{27}$.

En bon empiriste, William Hogarth entreprit, avec la publication de son Analyse de la Beauté (1753), de conceptualiser a posteriori les lignes de force d'une œuvre qu'il avait déjà pour l'essentiel réalisêe. Ce traité, l'un des premiers ouvrages d'esthétique formaliste de l'histoire de l'art, propose une tentative de définition de la beauté selon des principes empiristes, en accordant une place primordiale à l'observation et à une approche rationnelle de l'art.

Annoncé en mars 1752 dans le Covent Garden Journal de Fielding comme "un court traité in quarto, appelé L'Analyse de la Beauté, dans lequel les objets sont considérés sous un nouvel éclairage, tant en matière de forme que de couleur », l'ouvrage est accompagné de deux planches explicatives sur lesquelles Hogarth attire d'entrée de jeu l'attention : «Au premier coup d'œil le plan de cet ouvrage, ainsi que les planches qui l'accompagnent, ne paraîtront qu'un jeu de l'imagination, plus propre à égarer et à déconcerter qu'à amuser et à instruire, mais je me flatte que lorsqu'on aura examiné avec quelque attention les principes que j'y développe, qui tous sont fondés sur la nature, on ne les regardera plus comme indignes d'un plus mûr examen " ${ }^{28}$. Les premiers souscripteurs reçurent une gravure au symbolisme fort, Columbus breaking the Egg [FIG. 8], qui suggère un parallèle ambitieux entre la découverte de la "Ligne de Beauté " par Hogarth et celle de l'Amérique par Christophe Colomb : dans un cas comme dans l'autre, c'est bien d'un «nouveau monde » qu'il s'agit. De façon significative, l'un des premiers exemplaires

27. William Hazlitt, "On the Works of Hogarth », Lecture VII, Lectures on the English Comic Writers (Londres, 1819), p. 145 [ma traduction].

28. William Hogarth, ouvrage cité, p. 45. Dans l'annonce parue dans le General Advertiser (16 nov. 1752), Hogarth annonce que les deux gravures représenteront « une danse campagnarde et la cour d'un statuaire : qu'elles seront accompagnées d'une grande variété de figures, destinée à illustrer le nouveau système que l'ouvrage contient ». Sur la publication de cet ouvrage, ainsi que sur le contexte et les idées esthétiques anglaises de l'époque, on consultera la remarquable introduction de Ronald Paulson à la récente ré-édition du texte de Hogarth : William Hogarth, The Analysis of Beauty (New Haven and London, Yale University Press, 1997). 


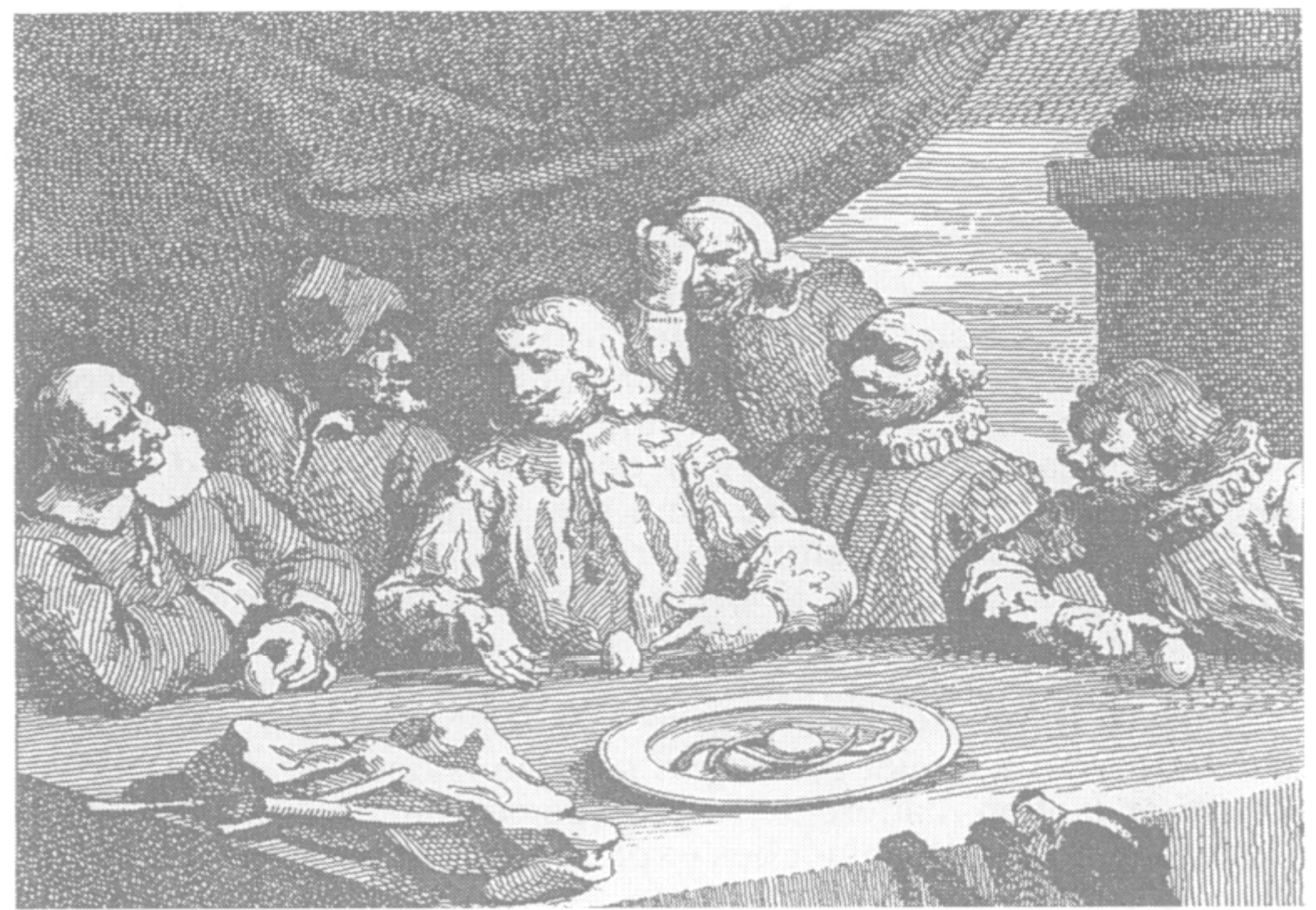

8. W. HOGARTH : Columbus breaking the Egg (1752).

de L'Analyse de la Beauté fut envoyé personnellement par Hogarth à Thomas Birch pour la bibliothèque de la Société Royale.

Rassemblant en un même mouvement les pans nationaliste et épistémologique de sa démarche, Hogarth cherche par son ouvrage à promouvoir la supériorité de l'observation directe sur la copie des Anciens, et appelle de ses vœux l'émergence d'une école de peinture (anglaise, bien sûr) qui trouverait ses sujets dans la Nature réelle et contemporaine (anglaise, de préférence), et non dans quelque reformulation fatiguée de modèles antiques ${ }^{29}$. Rejetant la vénération aveugle des doctrines antiques, il en dénonce les principes prétendument mystérieux, qui ont entraîné l'humanité « vers une sorte d'estime religieuse, voire de bigoterie, pour les ouvrages de l'antiquité » (chap. XI, ma traduction).

Pour lui, aucune statue de Vénus, jamais, ne surpassera la beauté d'une femme vivante, et ce lien entre le beau et le vivant,

29. Que la peinture anglaise ait pris ensuite son essor particulièrement dans les genres du paysage et du portrait n'est pas la moindre de ses réussites posthumes. 
cette recherche d'une «expérience des objets eux-mêmes dans la Nature " (Introduction, ma traduction), représente le cœur de son message. Cherchant dès la Préface à démystifier la beauté et son « je-ne-sais-quoi » prétendument inexplicable, Hogarth, au contraire, se pose en partisan d'une esthétique du « je-sais-quoi », fondée sur une croyance profonde en un monde compréhensible par la raison ${ }^{30}$. La perception de la beauté procède d'une observation attentive et sensorielle du monde et de ses "particulars", comme les appelle Locke, loin des présupposés élitistes ou abstraits. Réfutant les "superstitions", comme les propriétés «mystiques » du nombre d'or et autres échelles harmoniques qui prétendent donner une justification métaphysique à la suprématie de l'art des anciens, il veut promouvoir une «ligne de beauté » qui n'a rien de mystérieux, qui résulte "naturellement» de la combinaison d'un certain nombre de propriétés formelles objectives (convenance, variété, uniformité, régularité ou symétrie, simplicité ou netteté, complexité, quantité - en fait, les titres de tous les premiers chapitres du traité), et qu'il illustre dans ses planches par la représentation de pieds de chaise plus ou moins galbés [FIG. 4, no 50].

Constamment, son traité se réfère aux formes et aux objets du monde vivant, ses exemples et illustrations sont concrets [FIG. $4, \mathrm{n}^{\mathrm{os}} 1-7 ; 26 ; 33-34 ; 40-46$ ], ses démonstrations cherchent à être tangibles. Dans l'un des chapitres les plus remarquables, «Des Compositions avec la Ligne serpentine» (chap. X), il avance que, si le corps humain est beau, ce n'est pas pour une quelconque raison intellectuelle de proportion mathématique abstraite, mais simplement parce qu'il n'est pour l'essentiel composé que de lignes serpentines : «Â peine y a-t-il un os parfaitement droit dans toute la charpente du corps. Tous, pour ainsi dire, sont, non seulement courbés de différentes manières, mais tous ont aussi une espèce de torsion, laquelle est fort agréable dans quelques-uns; et les muscles qui s'y trouvent attachés, quoique de diverses formes, suivant les différents usages auxquels ils sont destinés, ont en général leurs fibres dans une direction serpentine $[\ldots]$ Les anatomistes qui connaissent la vérité de ce que j'avance prennent un grand plaisir à en distinguer les différentes beautés [...] On peut se convaincre qu'aucun objet n'offre autant de lignes serpentines que le corps humain, ce qui nous fournit

30. Le principal reproche fait à Hogarth, notamment par Reynolds, sera précisément cette volonté de ramener tout principe artistique à un savoir. 
une preuve de sa beauté supérieure, et montre que cette beauté n'est due qu'à ces lignes » (Analyse, p. 95-97). Recommandant lui aussi un véritable travail d'anatomiste, afin de scruter les moindres reliefs de la peau, les moindres contours des os, des nerfs et des fibres, Hogarth invite à comprendre les formes du vivant de l'intérieur, en se lovant mentalement, pour ainsi dire, dans le concave caché des objets (et des gens), car en « poussant ces recherches anatomiques un peu plus loin [...], on se trouvera insensiblement initié dans les principes qui constituent la grâce et la beauté d'un homme bien fait, et des plus admirables statues de l'antiquité : on apprendra également, par ce moyen, à connaître la cause du plaisir que leur vue cause à l'esprit sans qu'on puisse s'en rendre raison à soi-même » (Analyse, p. 99) ${ }^{31}$.

Vantant les mérites d'une esthétique dynamique, comparant la perception à une poursuite, il ne cesse de souligner l'importance, pour l'artiste puis pour le spectateur, de l'activité expérimentale de découverte. L'œuvre d'art, instrument d'optique, intensifie aussi le réel, l'ouvre au regard et en permet une véritable expérience. L'image artistique rejoint l'image scientifique au service d'un projet épistémologique où le poco piu, loin d'être l'évanescent et mystérieux secret de l'artiste, est le fruit concret de l'expérience et l'effet constamment renouvelé de lumineuses séries de causes.

\author{
FRÉDÉRIC OGÉE \\ Université Paris 7 - Denis Diderot
}

31. Dans les mêmes années, David Hume recommande une même association de l'artiste et de l'anatomiste : «Le peintre a besoin de l'anatomiste car, en plus d'un goût délicat et d'une bonne appréhension, il doit posséder une connaissance précise de la texture interne. [...] Aussi pénible que puisse apparaître cette recherche ou analyse interne, elle est dans une certaine mesure obligatoire à ceux qui ambitionnent de décrire avec succès les apparences externes évidentes de la vie et des mœurs. L'anatomiste présente à l'oil les objets les plus hideux et désagréables; mais sa science est utile au peintre, même pour dépeindre une Vénus ou une Hélène. Tout en utilisant les couleurs les plus riches de son art et en donnant à ses personnages les attitudes les plus gracieuses et les plus engageantes, l'artiste doit prêter aussi attention à la structure interne du corps humain, la position des muscles, la texture des os, et l'utilité et la forme de chacun des organes " (Philosophical Essays concerning Human Understanding (1748), ed. L. A. Selby-Bigge, rev. P. H. Nidditch (Oxford, 1975), I.5.10 (ma traduction]. 